\title{
ANÁLISE DA EFETIVIDADE DA TERAPIA COMUNITÁRIA INTEGRATIVA NA SAÚDE BIOPSICOSSOCIAL DE DIFERENTES POPULAÇÕES: UMA REVISÃO INTEGRATIVA
}

\author{
ANÁLISIS DE LA EFECTIVIDAD DE LA TERAPIA COMUNITARIA INTEGRATIVA \\ EN LA SALUD BIOPSICOSOCIAL DE DIFERENTES POBLACIONES: UNA \\ REVISIÓN INTEGRATIVA
}

\section{ANALYSIS OF THE EFFECTIVENESS OF INTEGRATIVE COMMUNITY THERAPY IN BIOPSYCHOSOCIAL HEALTH OF DIFFERENT POPULATIONS: AN INTEGRATIVE REVIEW}

\author{
Tissiane Paula ZEM IGESKI ${ }^{1}$ \\ Laremi Paixão da SILVA ${ }^{2}$ \\ Derivan Brito da SILVA ${ }^{3}$ \\ Milene Zanoni da SILVA ${ }^{4}$
}

RESUMO: A Terapia Comunitária Integrativa (TCI) pertence ao conjunto das Práticas Integrativas e Complementares (PICS). A TCI, enquanto ferramenta de cuidado em saúde promove um espaço de acolhimento ao sofrimento, sendo considerada uma tecnologia leve de promoção à saúde. Este estudo visa analisar a produção científica acerca a efetividade da TCI na saúde biopsicossocial de indivíduos e comunidades. Metodologicamente seguiram-se as seis etapas do método de Revisão Integrativa, tendo como pergunta norteadora: o que revela a produção científica no período de 2008 a 2018 acerca do impacto da TCI no cuidado em saúde? A partir dos 15 artigos científicos selecionados foi elaborada uma síntese acerca do tema: a efetividade da Terapia Comunitária Integrativa na saúde biopsicossocial. Este estudo sistematizou informações sobre a efetividade da TCI e constatou-se o impacto desta PICS na saúde mental e social, além de traçar o perfil e as lacunas da produção científica.

PALAVRAS-CHAVE: Terapia comunitária integrativa. Saúde mental. Atenção primária à saúde. Terapias complementares. Revisão integrativa.

RESUMEN: La Terapia Comunitaria Integrativa (TCI) pertenece al conjunto de Prácticas Integrativas y Complementarias (PICS). TCI, como herramienta asistencial, promueve un espacio de acogida del sufrimiento, siendo considerada una tecnología ligera para la promoción de la salud. Este estudio tiene como objetivo analizar la producción científica sobre

${ }^{1}$ Universidade Federal do Paraná (UFPR), Curitiba - PR - Brasil. Mestranda do Programa de Pós Graduação em Saúde Coletiva. ORCID: https://orcid.org/0000-0001-9974-6061. E-mail: tissizem@gmail.com

${ }^{2}$ Universidade Federal do Paraná (UFPR), Curitiba - PR - Brasil. Graduanda no Curso de Biomedicina. ORCID: http://orcid.org/0000-0003-2393-9195. E-mail: laremipaixaos@gmail.com

${ }^{3}$ Universidade Federal do Paraná (UFPR), Curitiba - PR - Brasil. Docente do Departamento de Terapia Ocupacional. Doutorado em Sociologia (UFPR). ORCID: https://orcid.org/0000-0002-0971-7441. E-mail: derivan to@hotmail.com

${ }^{4}$ Universidade Federal do Paraná (UFPR), Curitiba - PR - Brasil. Docente do Departamento de Saúde Coletiva. Doutorado em Saúde Coletiva (UEL). Vice-presidente da Associação Brasileira de Terapia Comunitária Integrativa (ABRATECOM). ORCID: http://orcid.org/0000-0002-1177-9668. E-mail: milenezanoni@gmail.com 
la efectividad de las TIC en la salud biopsicosocial de individuos y comunidades. Metodológicamente, se siguieron las seis etapas del método de Revisión Integrativa, con la pregunta orientadora: ¿qué revela la producción científica en el periodo de 2008 a 2018 sobre el impacto de las TIC en la atención de la salud? A partir de los 15 artículos científicos seleccionados, se elaboró una sintesis sobre el tema: la efectividad de la Terapia Comunitaria Integrativa en la salud biopsicosocial. Este estudio sistematizó información sobre la efectividad de las TIC y encontró el impacto de este PICS en la salud mental y social, además de rastrear el perfil y las brechas de la producción científica.

PALABRAS CLAVE: Terapia comunitaria integradora. Salud mental. Primeros auxilios. Terapias complementarias. Revisión integradora.

ABSTRACT: Integrative Community Therapy (ICT) is part of the Integrative and Complementary Practices (PICS). ICT, as a health care tool, promotes a welcoming space for suffering and is considered a lightweight technology for health promotion. This study aims to analyze the scientific production about the effectiveness of ICT in the biopsychosocial health of individuals and communities. Methodologically, the six stages of the Integrative Review method were followed, with the guiding question: what does the scientific production reveal in the period from 2008 to 2018 about the impact of ICT on health care? From the 15 scientific articles selected, a synthesis was elaborated on the theme: the effectiveness of Integrative Community Therapy in biopsychosocial health. This study systematized information on the effectiveness of ICT and verified the impact of this PICS on mental and social health, in addition to outlining the profile and gaps in scientific production.

KEYWORDS: Integrative community therapy. Mental health. Primary health care. Complementary therapies. Integrative review.

\section{Introdução}

A Terapia Comunitária Integrativa (TCI) foi criada em 1987 pelo Prof. Dr. Adalberto de Paula Barreto, psiquiatra e antropólogo e inicialmente desenvolvida pelo Departamento de Saúde Comunitária da Faculdade de Medicina do Ceará. Com origem no nordeste do Brasil, a TCI tem sido difundida tanto nacional quanto internacionalmente. Atualmente, a TCI está presente em países da Europa, África e Américas.

$\mathrm{Na}$ TCI "terapia" significa acolher, ser caloroso e cuidar das outras pessoas; "comunitária" expressa o que as pessoas têm em comum; e "integrativa" reporta aos saberes integrados das comunidades (BARRETO, 2019). Destarte, a TCI surge como um espaço de acolhimento para partilhar inquietações, problemas ou situações difíceis, bem como, para expressar alegrias, celebrar vitórias e histórias de superação (FERREIRA FILHA; LAZARTE; BARRETO, 2015; BARRETO, 2019). 
A TCI metodologicamente é sustentada por cinco pilares entrelaçados e articulados, a saber: pensamento sistêmico; teoria da comunicação; antropologia cultural; pedagogia de Paulo Freire; e resiliência (BARRETO, 2019).

O pensamento sistêmico permite perceber os problemas como pertencentes a uma rede complexa, formada por partes interdependentes. Assim, nas situações vivenciadas, cada indivíduo faz parte do problema e também da solução, pois os problemas e soluções são sistêmicos e estão interligados (BARRETO, 2019; DAMASCENO, 2011). A teoria da comunicação permite compreender que todo comportamento tem função de comunicar algo pela fala, pela escrita e por gestos (BARRETO, 2019; CARVALHO et al., 2019). Nessa perspectiva, por meio da comunicação, é possível ao indivíduo entender o mundo, transformar a própria realidade e a si mesmo (DAMASCENO, 2011).

A antropologia cultural auxilia no entendimento do conjunto de aspectos que os indivíduos herdam ao integrarem uma sociedade, permitindo que eles vivenciem e comportemse no mundo social (DAMASCENO, 2011). A pedagogia de Paulo Freire desperta para a análise crítica da realidade, com ênfase na valorização da sabedoria e experiência de vida dos indivíduos, com o objetivo de construir a consciência crítica (BARRETO, 2019).

Por fim, a resiliência significa voltar ao estado natural, com uma consciência baseada na estabilidade, no poder de adaptação e superação dos problemas do cotidiano (FERREIRA; FILHA; LAZARTE; DIAS, 2019).

Para o Ministério da Saúde (MS), a TCI é uma abordagem psicossocial, que permite construir redes solidárias, que potencializam os recursos e competências individuais e das comunidades, gerando autonomia (BRASIL, 2013). Recentemente, a TCI foi aprovada como uma Prática Integrativa e Complementar em Saúde (PICS) no Sistema Único de Saúde (SUS) pela Portaria nº 849/2017 (BRASIL, 2017). As PICS são cuidados em saúde com racionalidades próprias e cosmovisões singulares de acordo com a cultura e a década de origem (LUZ, 2003). Nos serviços do SUS a indicação das PICS, como forma de cuidado em saúde, ocorre predominantemente na Atenção Primária (BRASIL, 2020).

O número de estudos sobre a TCI no Brasil revela lacunas de informações acerca desta PICS, como por exemplo, o seu impacto em diferentes populações e contextos. Neste momento de intenso debate acerca da manutenção ou não das PICS no SUS, incluindo a TCI (SAVARIS, 2018), é iminente a necessidade de produzir evidências sólidas para subsidiarem o processo decisório na esfera pública. Desta forma, esta investigação teve como objetivo analisar a produção científica acerca a efetividade da TCI na saúde biopsicossocial de indivíduos e comunidades, no período entre 2008 e 2018. 


\section{Método}

A trajetória metodológica selecionada neste estudo foi a Revisão Integrativa (RI), por viabilizar a condensação de resultados obtidos em pesquisas sobre um tema, de forma sistemática, ordenada e abrangente, e visa, entre outros objetivos, a análise metodológica dos estudos sobre o tema em particular (MENDES, 2008), no caso aqui a: análise da efetividade da Terapia Comunitária Integrativa na saúde biopsicossocial.

Seguindo as seis etapas da Revisão Integrativa, proposta por Mendes (2008), na primeira etapa identificou-se o tema da pesquisa, supracitado, e elegeu-se a seguinte pergunta norteadora da pesquisa: o que revela a produção científica no período de 2008 a 2018 acerca do impacto da TCI no cuidado na saúde biopsicossocial de indivíduos e comunidades?

Na segunda etapa, considerando que o termo "terapia comunitária" não é um descritor indexado, foram utilizadas como palavras-chaves: "terapia comunitária" OR "terapia comunitária integrativa" OR "integrative community therapy" OR "community therapy", e estabelecidos os seguintes critérios de inclusão: artigos publicados no período de 2008 a 2018, nos idiomas português, espanhol e inglês e texto completo. Foram utilizados como critério de exclusão os artigos que não apresentaram fundamentação teórica em acordo com metodologia proposta por Adalberto Barreto, descrita em sua obra "Terapia Comunitária: passo a passo".

Na terceira etapa, para a extração de informações dos artigos incluídos no estudo, foram definidas as seguintes variáveis: população estudada; cenário/serviço onde ocorreu o estudo; região do Brasil onde o estudo foi realizado; tipo de pesquisa e abordagens; instrumentos utilizados; enfoque dado à pesquisa; referenciais de análise; os impactos alcançados; área de formação dos pesquisadores; revista/caderno científico e seu respectivo qualis e financiamento científico.

$\mathrm{Na}$ quarta etapa elaboraram-se tabelas que permitissem a ordenação, classificação e avaliação das informações extraídas dos artigos. A partir da abordagem quantitativa, os dados foram selecionados e analisados estatisticamente através de cálculos percentuais simples.

$\mathrm{Na}$ quinta etapa buscou-se analisar as informações visando à interpretação dos resultados. A avaliação das informações foi realizada por meio da epidemiologia descritiva, com frequências absolutas e relativas.

Por fim, na sexta etapa foi buscou-se a um diálogo entre as variáveis selecionadas na terceira etapa, em prol da síntese do conhecimento acerca do impacto da TCI no cuidado em saúde. A classificação do impacto da TCI foi categorizada como social, mental/emocional, biológico e espiritual, tendo em vista os efeitos percebidos pelos participantes das rodas. 
Esclarecemos que o resultado da quarta, quinta e sexta etapa compõem os resultados e as considerações finais deste trabalho.

\section{Resultados}

Com base na pergunta norteadora e estratégia de busca, foram selecionados 23 artigos da BVS, 07 da Pubmed e 06 da Scielo, totalizando 36 artigos, sendo que 05 (13,8\%) deles eram duplicados. Dos 31 artigos encontrados, apenas 15 (48,4\%) cumpriram os critérios de inclusão.

O número de artigos encontrado e publicados com o tema da efetividade da TCI na saúde é muito baixo comparado ao montante de publicações a respeito de outras PICS, como medicina tradicional chinesa, acupuntura ou fitoterapia. São diversos os desafios quando se trata de pesquisa no campo da TCI, seja por suas especificidades históricas, sociais e metodológicas seja pelo cenário brasileiro quando se trata de fomentar pesquisas no âmbito das PICS.

De um modo geral, apesar do crescimento de pesquisas em PICS no Brasil nas últimas décadas, ainda é necessário ampliar os estudos. De acordo com Tesser, Sousa e Nascimento (2018), na base de dados Bireme (Centro Latino-Americano e do Caribe de Informação em Ciências da Saúde), de 7.243 publicações científicas sobre PICS entre os anos de 2006 e 2016, somente 3\% (285) têm afiliação institucional brasileira. Este fato pode ser justificado pelo reduzido financiamento para pesquisa nesta temática.

De acordo com Tesser, Sousa e Nascimento (2018, p. 182) em 2013 a pesquisa em PICS teve seu primeiro edital específico, único em 10 anos de PNPICS (2006). O edital de Chamada MCTI/CNPq/MS - SCTIE - Decit No 07/2013 - Política Nacional de Práticas Integrativas e Complementares (PICS) no SUS, contemplou pesquisadores de 10 estados, contudo nenhuma pesquisa tinha como escopo a terapia comunitária, pois se privilegiou as PICS instituídas pela PNPICS, tal como a acupuntura (26\%) e fitoterapia (21\%).

Dentre os desafios da pesquisa em TCI, foi percebida a ausência de estudos com financiamento institucional para sua realização nos artigos analisados. Para Dudziak (2018) as principais agências de financiamento da pesquisa - CNPq e Capes - enfrentam problemas com a escassez de recursos. A partir de pesquisa sobre o fomento de estudos no Brasil, dos anos de 2011 a 2018, o estado brasileiro com maior produtividade e custeio foi São Paulo, seguido do Rio de Janeiro, Minas Gerais, Rio Grande do Sul e Paraná. Os três órgãos que mais investiram em pesquisa no Brasil foram o CNPq, CAPES e FAPESP. As áreas de mais destaque quanto ao 
subsídio foram a Biologia Molecular e Bioquímica, Ciências das Plantas/Biologia Vegetal, Ciência dos Materiais, Farmácia e Farmacologia.

Esses dados comprovam a necessidade já relatada de recursos econômicos para grupos de pesquisadores de outras regiões do Brasil, bem como em outras áreas - tal como a promoção à saúde -, pois atualmente as pesquisas em TCI vêm sendo realizadas por aspiração pessoal e sem apoio orçamentário.

Além disso, a TCI que é uma PICS que nasceu na e para as comunidades, e tem se fortalecido como ferramenta de transformação social e de promoção da saúde, onde - apesar de até 2011 terem sido capacitados mais 30 mil terapeutas comunitários no contexto nacional (REIS, 2017) - são poucos os terapeutas comunitários que tem interlocução com universidades, dificultando o estabelecimento de redes e parcerias para produção científica.

Das 29 PICS institucionalizadas no SUS, apenas a TCI tem a origem genuinamente brasileira, sendo uma das mais recentes se comparada às outras práticas, que são milenares, tais como medicina tradicional chinesa, medicina ayurveda ou fitoterapia. Este fato pode justificar o motivo pelo qual a TCI ainda é um tema livre e não um descritor em saúde (DECS, 2020).

Conceitualmente, descritores em ciências da saúde (DeCS) são vocabulários necessários para descrever, organizar e prover acesso à informação na área da saúde. Seu uso permite ao pesquisador recuperar a informação com o termo exato utilizado para descrever o conteúdo daquele documento científico. No caso da TCI, que ainda não é um DeCS, os artigos publicados nesta área têm risco de não serem encontrados e, portanto, nem citados, perdendo-se informações (BRANDAU; MONTEIRO; BRAILE, 2005).

Figura 1 - Tabela da caracterização dos estudos publicados acerca da efetividade da TCI na saúde, 2008-2018

\begin{tabular}{|c|c|c|c|c|c|}
\hline Categorias & $\mathbf{N}$ & $\%$ & Categorias & $\mathbf{N}$ & $\%$ \\
\hline Idioma & & & Enfoque da pesquisa & 15 & 100 \\
\hline Português & 15 & 100 & Promoção da saúde & 9 & 60 \\
\hline Inglês & 0 & 0 & Educação permanente & 1 & 6,6 \\
\hline Espanhol & 0 & 0 & Reabilitação & 5 & 33,3 \\
\hline Período & & & $\begin{array}{l}\text { Área de formação dos } \\
\text { pesquisadores }\end{array}$ & 49 & 100 \\
\hline $2008-2013$ & 7 & 46,6 & Enfermagem & 40 & 81,6 \\
\hline 2014-2018 & 8 & 53,3 & Medicina & 3 & 6,1 \\
\hline Regiões do Brasil & & & Psicologia & 2 & 4 \\
\hline Nordeste & 11 & 73,3 & Terapeutas comunitárias & 2 & 4 \\
\hline Centro- Oeste & 3 & 20 & Outros & 2 & 4 \\
\hline Sudeste & 1 & 6,6 & Estados do Brasil & & \\
\hline Sul & 0 & 0 & Paraíba & 7 & 46,6 \\
\hline Norte & 0 & 0 & Mato Grosso & 3 & 20 \\
\hline \multicolumn{3}{|c|}{ Financiamento de pesquisa científica } & Ceará & 2 & 13,3 \\
\hline Não & 15 & 100 & Rio Grande do Norte & 2 & 13,3 \\
\hline Sim & 0 & 0 & Rio de Janeiro & 1 & 6,6 \\
\hline
\end{tabular}

Fonte: elaborado pelos autores 
Das publicações a serem encontradas a partir dos artigos selecionados e do escopo da investigação $(\mathrm{n}=15)$, a figura 1 expõe que a maioria dos estudos foi realizado de 2014 a 2018 correspondendo a 53,3\%, justificando-se pela inserção da Terapia Comunitária Integrativa na PNPIC a partir de portaria publicada em 2017.

A região nordeste obteve $73,3 \%$ dos artigos publicados, sendo $46,6 \%$ somente no estado da Paraíba. Este estado apresenta o Grupo de Estudos e Pesquisa em Saúde Mental Comunitária-GEPSMEC, que se dedica aos estudos da TCI com maior profundidade, impulsionando as produções acadêmicas a partir de pesquisas realizadas na graduação e pósgraduação na Universidade Federal da Paraíba - UFPB (FILHA et al., 2019).

Quanto ao enfoque dado aos estudos, $60 \%$ é voltado à promoção da saúde, fato que pode ser justificado pela natureza da TCI e suas características. Neste artigo o conceito de promoção da saúde coaduna como um processo de aprendizagem da comunidade para a melhoria da qualidade de vida e saúde, através da autonomia, identificando as aspirações e satisfazendo as necessidades (BRASIL, 2002). Nesta perspectiva, a TCI pode ser considerada como recurso de promoção à saúde pela criação de espaços de escuta e inclusão no território, valorizando a diversidade e resgatando a cultura popular e história de vida dos indivíduos, fortalecendo vínculos e redes de apoio. Destaca-se como importante instrumento de cuidado e de extrema relevância para a consolidação de várias políticas públicas, principalmente, como a de práticas integrativas e complementares, atenção básica e de saúde mental (AZEVEDO et al., 2013).

A área de formação dos pesquisadores com maior destaque nos estudos de intervenção da TCI foi a enfermagem com 81,6\% dos artigos publicados. Para Backes (2012), o enfermeiro tem a função decisiva e proativa para a identificação das necessidades de cuidado da população, bem como na promoção e proteção da saúde dos indivíduos nas suas necessidades. Há um elo entre a promoção da saúde, atenção primária e enfermagem nos resultados desta revisão integrativa, expondo o predomínio de publicações em seus campos de atuação, especialmente na Estratégia de Saúde da Família (ESF), na qual, esse ator, compõe a equipe juntamente com os profissionais médicos generalistas/saúde da família e comunidade, dentistas, técnicos de enfermagem e de saúde bucal, além dos agentes comunitários de saúde e de endemias.

A TCI é muito utilizada por esses profissionais, pois viabiliza alcançar os objetivos almejados no cuidado com os usuários, além do reconhecimento pelos próprios como ferramenta de autocuidado (GUIMARÃES; SOARES; SANTOS, 2019).

$\mathrm{O}$ fato de os pesquisadores terem formação na área de enfermagem justifica os dados apresentados na figura 2 , que se refere às revistas que mais têm publicado artigos $-60 \%$ são 
periódicos nesta área de conhecimento. As revistas com maior aceitabilidade na temática da TCI, no período analisado, foram a Revista Eletrônica de Enfermagem (33,3 \%) e a Revista Gaúcha de Enfermagem (22,2\%). Na análise também foi observado que as revistas com maior número de publicações foi a de Qualis B2 (53,3\%) e Qualis B1 com 20\%.

Figura 2 - Tabela da caracterização das revistas científicas e da qualidade dos periódicos analisados sobre a TCI na saúde, 2008-2018

\begin{tabular}{lccc}
\hline Revistas de Publicação & Qualis & N & $\%$ \\
\hline Revistas na área da Enfermagem & & 9 & $\mathbf{6 0}$ \\
\hline Revista Eletrônica de Enfermagem & B2 & 3 & 33,3 \\
Revista Gaúcha de Enfermagem & B1 & 2 & 22,2 \\
Revista Latino Americana de Enfermagem & A1 & 1 & 11,1 \\
Revista de Enfermagem da UFSM/RS & B2 & 1 & 11,1 \\
Revista Brasileira de Enfermagem & A2 & 1 & 11.1 \\
Revista Texto e Contexto/Enfermagem & A2 & 1 & 11,1 \\
Outras & & $\mathbf{6}$ & $\mathbf{4 0}$ \\
Revista de Pesquisa: Cuidado é fundamental online & B2 & 3 & 50 \\
Caderno de Saúde Pública & A3 & 1 & 16,6 \\
Revista de Atenção à Saúde & B2 & 1 & 16,6 \\
Revista Eletrônica de Saúde Mental - Álcool e Drogas & B1 & 1 & 16,6 \\
\hline
\end{tabular}

Fonte: elaborado pelos autores

Na figura 3 observou-se a caracterização das publicações de acordo com as questões metodológicas nos diversos trabalhos. A Atenção Primária em Saúde (APS) é o cenário de pesquisa onde houve maior prevalência das rodas de TCI (50\%). Segundo o site do Ministério da Saúde, a ESF e as equipes do Núcleo Ampliado de Saúde da Família e Atenção Primária (NASF-AP) concentram 78\% das PICS realizadas no SUS, destacando-se em relação aos outros níveis. No estudo de Tesser, Souza e Nascimento (2018), que analisou dados do PMAQ-AB, a TCI foi a quarta PICS $(10,4 \%)$ mais ofertada pelas equipes de APS no Brasil, estando atrás apenas das práticas corporais da Medicina tradicional Chinesa $(16,6 \%)$, plantas medicinais e fitoterapia $(14,9 \%)$ e acupuntura $(12,7 \%)$. 
Figura 3 - Tabela da caracterização dos estudos analisados de acordo com as questões metodológicas dos artigos publicados sobre a efetividade da TCI na saúde, entre 2008-2018

\begin{tabular}{|c|c|c|c|c|c|}
\hline Categorias & $\mathbf{N}$ & $\%$ & Categorias & $\mathbf{N}$ & $\%$ \\
\hline $\begin{array}{l}\text { Quantitativo de artigos por } \\
\text { população alvo }\end{array}$ & 15 & 100 & $\begin{array}{l}\text { Quantitativo da população alvo } \\
\text { participante das rodas de TCI }\end{array}$ & 995 & 100 \\
\hline Usuários e profissionais da APS & 5 & 33,3 & Usuários e profissionais da APS & 354 & 35,6 \\
\hline $\begin{array}{l}\text { Familiares / usuários de CAPS e } \\
\text { Comunidade terapêutica }\end{array}$ & 3 & 20 & $\begin{array}{l}\text { Familiares / usuários de CAPS e } \\
\text { Comunidade terapêutica }\end{array}$ & 336 & 33,7 \\
\hline Outros & 3 & 20 & Idosos & 279 & 28 \\
\hline Idosos & 2 & 13,3 & Universitários & 10 & 1 \\
\hline Universitários & 1 & 6,7 & Outros & 9 & 0,9 \\
\hline Mulheres & 1 & 6,7 & Mulheres & 7 & 0,7 \\
\hline Cenários de pesquisa & 10 & & Abordagens do estudo & 15 & \\
\hline Atenção Primária em Saúde & 5 & 50 & Qualitativa & 13 & 86,6 \\
\hline CAPS e com. terapêutica & 3 & 30 & Quali-Quantitativa & 2 & 13,3 \\
\hline Universidade & 2 & 20 & Quantitativa & 0 & 0 \\
\hline Tipos de estudo & 19 & & Tipos de instrumentos utilizados & 21 & \\
\hline Exploratório & 6 & 27,2 & Entrevistas & 8 & 38,1 \\
\hline Documental & 5 & 22,7 & Documentos & 4 & 19 \\
\hline Descritivo & 3 & 13,6 & Diário de campo & 4 & 19 \\
\hline Relato de experiência & 2 & 9 & Filmagem & 2 & 9,5 \\
\hline História oral & 2 & 9 & Questionários & 2 & 9,5 \\
\hline Etnográfico & 1 & 4,5 & $\begin{array}{l}\text { Teste de Associação livre de } \\
\text { Palavras -TALP }\end{array}$ & 1 & 4,8 \\
\hline Experimental & 0 & 0 & Referencial de análise & 15 & \\
\hline Temporalidade do estudo & 15 & & Análise de conteúdo & 10 & 66,7 \\
\hline Retrospectivo & 11 & 73,3 & Não informado & 2 & 13,3 \\
\hline Transversal & 2 & 13,3 & Descrição estatística & 2 & 13,2 \\
\hline Prospectivo & 2 & 13,3 & Triangulação de métodos & 1 & 6,6 \\
\hline
\end{tabular}

Fonte: elaborado pelos autores

A relevância da TCI como recurso terapêutico de cuidado integral no SUS pode ser justificado considerando a magnitude de sofrimento mental na APS (MENEZES et al., 2019) e transtornos mentais leves (9 a 12\%) além da prevalência de transtornos mentais severos e persistentes (3\%), que necessitam de cuidados contínuos (BRASIL, 2010; SILVEIRA; VIEIRA, 2009). De acordo com a Política Nacional de Saúde Mental, esta população deve ser acompanhada na APS, que promove espaços de atenção à saúde no território, próximo a rede familiar, social e cultural do indivíduo (BRASIL, 2010). Outro aspecto a salientar é que apesar da inserção da TCI em diversas áreas, tais como justiça, educação e assistência social, foi na 
área da saúde que, em 2017, a TCI se tornou uma política pública, face seus benefícios para a promoção da saúde, prevenção de doenças e reabilitação (CEZÁRIO et al., 2015).

Como modelo de experiência exitosa na APS, cabe reconhecer o estudo de Corrêa e Silveira (2015), acerca do processo de implementação da rede de atenção psicossocial (RAPS) no município de Santa Terezinha de Itaipu - PR, na qual uma das ações de cuidado em saúde mental era a implantação da Terapia Comunitária Integrativa na APS. Para alcançar tal objetivo houve a formação em Terapia Comunitária Integrativa dos profissionais da APS, ofertada pela parceria entre Itaipu Binacional, Universidade de Integração Latino Americana (UNILA) e Universidade Federal do Paraná (UFPR), capacitando 70 terapeutas comunitários do Brasil e Paraguai.

Com o investimento em educação permanente e da organização da RAPS municipal, a prática das rodas de TCI foi estabelecida para os usuários da APS, que foram identificados e estratificados para que recebessem a atenção à saúde necessária às suas demandas. Por meio dessa estratégia resultou-se na eliminação da fila de espera por atendimentos individuais na psicologia e psiquiatria nas Unidades Básicas de Saúde, aumentando a resolutividade da APS com alívio do sofrimento mental dos indivíduos (CORRÊA; SILVEIRA, 2015).

A respeito das abordagens metodológicas dos estudos (figura 2), a mais frequente foi a qualitativa $(86,6 \%)$, remetendo-se ao recurso da experiência e da vivência sentida pelo indivíduo e pelo coletivo na TCI, sendo uma das premissas relevantes para entender a sua contribuição à saúde das populações.

Ao considerar os trabalhos analisados, não foi encontrado nenhum artigo de caráter experimental envolvendo a TCI, necessitando avançar em pesquisas epidemiológicas com maior nível de evidência científica. A carência e a importância de mais pesquisas neste teor justificam-se pelo caráter complementar à pesquisa qualitativa, promovendo um olhar mais geral e amplodo objeto de estudo, enfatizando o raciocínio dedutivo, as regras da lógica e os atributos mensuráveis da experiência humana.

O estudo exploratório foi utilizado em 6 artigos (27,2\%), já esperado pelo seu caráter incipiente objetivando maior familiaridade com o problema, aspirando sua compreensão e a construção de hipóteses, já que são poucas as pesquisas acerca do tema estudado.

Os artigos retrospectivos representaram $73,3 \%$, e como instrumentos de coleta de dados, as entrevistas estiveram presentes em $34,8 \%$ dos estudos. A escolha do referencial de análise dos resultados foi a análise de conteúdo, a qual possui caráter interpretativo e investigativo das comunicações, correspondendo a $66,7 \%$ dos artigos. 
Figura 4 - Tabela da caracterização dos impactos da TCI a partir dos artigos selecionados para revisão integrativa sobre TCI na saúde entre 2008 a $2018(n=48)$

\begin{tabular}{|c|c|c|}
\hline Categorias & $\mathbf{N}$ & $\%$ \\
\hline Impactos da TCI & 48 & 100 \\
\hline Impacto mental/emocional & 28 & 58,3 \\
\hline Alivio do so frimento & 12 & 25 \\
\hline Empederamente, autoconfiança e autoestima & 7 & 14,5 \\
\hline Promoção da autonomia & 4 & 8,3 \\
\hline Construção da resiliência & 3 & 6,2 \\
\hline Espaço para expressar sentimentos e reflexão & 2 & 4,2 \\
\hline Impacto social & 15 & 31,2 \\
\hline Fortalecimento de vínculos & 2 & 18,7 \\
\hline Espaço de partilha & 6 & 12,5 \\
\hline Impacto biológico & 3. & 6,2 \\
\hline Prevenção de doenças & 2. & 4,2 \\
\hline Promoção da reabilitação psicossocial & 1 & 2,1 \\
\hline Impacto espiritual & 2 & 4,2 \\
\hline $\begin{array}{l}\text { Estímulo ao suporte emocional e superação de dificuldades a partir do } \\
\text { resgate da esperança e fé }\end{array}$ & 2 & 4,2 \\
\hline
\end{tabular}

Fonte: elaborado pelos autores

A maior contribuição da TCI quanto aos seus impactos foi na dimensão emocional/mental da saúde das pessoas e comunidades com 58,3\% do total, sendo o alívio do sofrimento (25\%), o fortalecimento do empoderamento e o resgate de autoestima e autoconfiança $(14,5 \%)$ os resultados mais frequentes.

Para Cassel (1982), o sofrimento pode ser considerado um estado de aflição severa, decorrente de acontecimentos que ameaçam a integridade da pessoa, exigindo consciência de si, envolvendo as emoções com efeitos nas relações pessoais dos indivíduos e nas dimensões social, familiar, física, emocional e espiritual. São fontes geradoras de emoções negativas as doenças, conflitos, desemprego, problemas financeiros, desigualdades sociais, violências e perdas, as quais podem gerar sintomas orgânicos, angústias e problemas psicossociais.

Logo, o que a TCI propicia é o manejo do sofrimento de forma culturalmente sensível e socialmente relevante, por um lado desmedicalizando os serviços de saúde, reconhecendo o impacto deletério dos determinantes sociais no processo saúde-doença e por outro, valorizando 
a dimensão integral do indivíduo, com seus recursos internos, tais como confiança e estima de si mesmo (14,5\%), resiliência $(6,2 \%)$, empoderamento e autonomia $(8,3 \%)$.

Assim, a TCI atua positivamente no adoecimento provocado pelo estresse crônico e desesperança que Barreto intitula de Síndrome da precariedade psíquica, que é a perda da confiança em si, nos outros e no futuro, estimulando a transformação das carências em competências e dos sofrimentos em resiliência e superações (FILHA; LAZARTE; BARRETO, 2015).

O impacto social foi observado em $31,2 \%$ dos artigos, sendo evidente no fortalecimento de vínculos coletivos, fundamental nos espaços de partilha conectando os indivíduos com a comunidade e cultura, valorizando o sentido de pertencer a um grupo a partir da construção de laços relacionais e da consciência social, com ações transformadoras de solidificação das redes de apoio (MOURA et al., 2017).

As repercussões biológicas apareceram em 6,2\% dos estudos, através da prevenção de doenças e da promoção da reabilitação psicossocial. Já os impactos espirituais corresponderam a $4,1 \%$ dos resultados, considerando o apoio por meio do resgate da fé e da esperança.

\section{Considerações finais}

Este estudo sistematizou informações sobre a efetividade da TCI e constatou-se o impacto desta PICS principalmente na saúde mental e social, além de traçar o perfil e as lacunas da produção científica com este escopo em TCI.

A terapia comunitária é uma prática integrativa que conquistou espaço como política pública na saúde no Brasil por seus impactos na promoção da saúde e prevenção de doenças, a partir de metodologia acessível e participativa que, por meio da palavra e histórias de vida, valoriza recursos pessoais e culturais - tais como confiança em si mesmo, resgate da autoestima, autonomia, fortalecimento de vínculos - com baixos custos, na contramão do modelo biomédico que medicaliza a vida e do sofrimento.

$\mathrm{Na}$ pesquisa tem-se um campo fértil para a produção do conhecimento na área, em especial, em pesquisas mistas, estudos de caso e estudos epidemiológicos, sendo de extrema importância a consolidação de parcerias, instituições e pesquisadores em TCI, com perspectivas de apoio e financiamento, para ampliar as evidências em saúde, que são fundamentais para as tomadas de decisões na gestão em saúde e que levam ao fortalecimento da TCI como política pública. 
Neste momento histórico e político de desmonte da estrutura pública no Brasil, incluindo o Sistema Único de Saúde e suas Políticas de Saúde Mental e de APS, a TCI como uma PICS de abordagem grupal genuinamente brasileira, se consolida, apesar do contexto, como uma ferramenta de cuidado em saúde mental, empoderamento, construção de vínculos que efetiva o direito e acesso à saúde à população, em especial os mais vulneráveis, de forma universal, equitativa e integral.

\section{REFERÊNCIAS}

AZEVEDO, E. B. de et al. Pesquisas brasileiras sobre terapia comunitária integrativa.

Revista Brasileira de Pesquisa em Saúde, Vitória, v. 15, n. 3, p. 114-120, jul./set. 2013.

BACKES, D. S. et al. O papel profissional do enfermeiro no Sistema Único de Saúde: da saúde comunitária à estratégia de saúde da família. Ciência e Saúde Coletiva, Rio de Janeiro, v. 17, n. 1, 2012. Disponível em: www.scielo.br/scielo.php?script=sci_arttext\&pid=S141381232012000100024\&1ng=en\&nrm=iso. Acesso em: 11 mar. 2020.

BARRETO A. P. Terapia comunitária: passo a passo. 5. ed. Fortaleza: LCR, 2019.

BRANDAU, R.; MONTEIRO, R.; BRAILE, D. M. Importância do uso correto dos descritores nos artigos científicos. Revista Brasileira Cir Cardiovascular, São José do Rio Preto, v. 20, n. 1, p. 8-9, mar. 2005.

BRASIL. Ministério da Saúde. Secretaria de Políticas de Saúde. Projeto Promoção da Saúde. As Cartas da Promoção da Saúde. Brasília: Ministério da Saúde, 2002.

BRASIL. Ministério da Saúde. Secretaria de Atenção à Saúde. Departamento de Atenção Básica. Diretrizes do NASF: Núcleo de Apoio a Saúde da Família. Brasília: Ministério da Saúde, 2010. 152 p. (Caderno de Atenção Básica, n. 27)

BRASIL. Ministério da Saúde. Saúde mental. Cadernos de Atenção Básica, n. 34. Brasília: Ministério da Saúde, 2013. Acesso em: 22 jan 2020. Disponível em:

http://189.28.128.100/dab/docs/portaldab/publicacoes/caderno_34.pdf. Acesso em: 15 jul. 2020.

BRASIL. Ministério da Saúde. Portaria n. 849, de 27 de março de 2017. Disponível em: http://bvsms.saude.gov.br/bvs/saudelegis/gm/2017/prt0849_28_03_2017.html. Acesso em: 26 jan. 2020.

CARVALHO, M. A. P. et al. A terapia comunitária integrativa enquanto intervenção psicossocial avançada: uma trama de aparelhamento comunitário. In: FERREIRA FILHA, M. de O.; LAZARTE, R.; DIAS, M. D. (Org.). Terapia comunitária integrativa e a pesquisa ação/intervenção: estudos avaliativos. João Pessoa: Editora UFPB, p. 33-49, 2019.

CASSEL, E. J. A natureza dos sofrimentos e os objetivos da medicina. N Engl J Med, Massachusetts, v. 306, n. 11, p. 639-645, mar. 1982. 
CEZÁRIO, P. F. O. et al. Integrative Community Therapy and its Benefits for Primary Care: an Integrative Review. International Archives of Medicine, v. 8, n. 267, p. 1-9, 2015.

CORREAA, R. S. Uma análise estratégica do processo de implementação da rede de Atenção em saúde mental no município de Santa Terezinha de ITAIPU: as Rodas de Terapia Comunitária Integrativa como um instrumento de Educação Permanente em Saúde. 2015. 35. Monografia (Trabalho de Conclusão do Curso de Especialização Gestão do Trabalho e da Educação na Saúde) - Escola de Saúde Pública do Paraná/Escola Nacional de Saúde Pública Sergio Arouca/FIOCRUZ, Curitiba, 2015.

DAMASCENO, R. C. Terapia comunitária integrativa: construção de um espaço de escuta para os trabalhadores da saúde da atenção básica. 2011. 99 f. Dissertação (Mestrado) Universidade Federal do Rio Grande do Norte, Natal, 2011.

DECS. Descritores em Ciências da Saúde. 22. ed. São Paulo: BIREME, OPAS, OMS, 2020. Disponível em: http://decs.bvsalud.org. Acesso em: 07 mar. 2020.

DUDZIAK, E.A. Quem financia a pesquisa brasileira? Um estudo InCites sobre o Brasil e a USP. São Paulo: SIBiUSP, 2018. Disponível em: https://zenodo.org/record/1317042. Acesso em: 10 mar. 2020.

FERREIRA FILHA, M. de O.; LAZARTE, R.; BARRETO, A. de P. Impacto e tendências do uso da Terapia Comunitária Integrativa na produção de cuidados em saúde mental. Revista Eletrônica De Enfermagem, v. 17, n. 2, p. 172-173, abr./jun. 2015.

FERREIRA FILHA, M. de O.; LAZARTE, R.; DIAS, M. D. (Orgs). Terapia comunitária integrativa e a pesquisa ação/intervenção: estudos avaliativos. João Pessoa: Editora UFPB, 2019.

LUZ, M. T. Racionalidades médicas e terapêuticas alternativas. Rio de Janeiro: UERJ/IMS, 1993.

MENDES, K. D. S.; SILVEIRA, R. C. C. P.; GALVÃO, C. M. Revisão integrativa: método de pesquisa para a incorporação de evidências na saúde e na enfermagem. Texto Contexto Enferm., Florianópolis, v. 17, n. 4, p. 758-764, out./dez. 2008.

MENEZES, A. L. do A. et al. Narrativas de sofrimento emocional na Atenção Primária: contribuições para uma abordagem integral culturalmente sensível em Saúde Mental Global. Interface, Botucatu, v. 23, 2019. Disponível em:

http://www.scielo.br/scielo.php?script=sci_arttext\&pid=S141432832019000100222\&lng=en\&nrm=iso. Acesso em: 21 abr. 2020.

MOURA, S. G. et al. Representações sociais sobre terapia comunitária integrativa construídas por idosos. Revista Gaúcha de Enferm. Porto Alegre, v. 38, n. 2, p. 1-6. jun. 2017.

REIS, M. L. de A. Quando me encontrei, voei: o significado da capacitação em terapia comunitária integrativa na vida do terapeuta comunitário. Porto Alegre: Caifcom, 2017.

SAVARIS, L. E. O cuidado integral na saúde coletiva: interfaces da dimensão religiosa/espiritual, práticas integrativas e complementares e saúde mental no Sistema Único 
de Saúde de Curitiba. 2018. 117 f. Dissertação (Mestrado em Saúde Coletiva) - Universidade Federal do Paraná, Curitiba, 2018.

SILVEIRA, D. da; VIEIRA, A. L. S. Saúde mental e atenção básica em saúde: análise de uma experiência no nível local. Ciência e Saúde Coletiva, Rio de Janeiro, v. 14, n. 1, p. 139-148, fev. 2009.

TESSER, C. D.; SOUSA, I. M. C. de; NASCIMENTO, M. C. do. Análise da efetividade da Terapia Comunitária Integrativa na saúde biopsicossocial de diferentes populações: uma revisão integrativa. Revista Saúde em Debate, Rio de Janeiro, v. 42, n. 1, p. 174-188, set. 2018.

\section{Como referenciar este artigo}

ZEM IGESKI, T. P.; SILVA, L. P. da; SILVA, D. B. da; SILVA, M. Z. da. Análise da efetividade da Terapia Comunitária Integrativa na saúde biopsicossocial de diferentes populações: uma revisão integrativa. Temas em Educ. e Saúde, Araraquara, v. 16, n. esp. 1, p. 271-285, set., 2020. e-ISSN 2526-3471. DOI: https://doi.org/10.26673/tes.v16iesp.1.13737

Submetido em: 20/05/2020

Revisões requeridas: $30 / 05 / 2020$

Aprovado em: 25/08/2020

Publicado em: 30/09/2020 